\title{
Very Large Solid Angle Windowless SDD Applications for Nanostructure and Semiconductor Applications
}

\author{
S. Bhadare ${ }^{1}$, P. Philips ${ }^{2}$, T. Paulauskas ${ }^{2}$, R. Klie ${ }^{2}$, N. Rowlands ${ }^{3}$ and A. Nicholls ${ }^{2}$ \\ 1. Oxford Instruments NanoAnalysis, Halifax Road, High Wycombe, HP12 3SE, UK. \\ 2. University of Illinois 845 W Taylor Street, Chicago, IL 60607. \\ 3. Oxford Instruments NanoAnalysis, 300Baker Avenue, Suite 150, Concord, MA 1742.
}

The use of SDDs gives numerous advantages over conventional $\mathrm{Si}(\mathrm{Li})$ detectors. Large solid angle allows data to be acquired faster in low signal regimes, and high count rate situations are handled more easily due to inherent lower noise and excellent energy resolution [1]. Due to lower noise these detectors may also be operated at near room temperatures and only Peltier type cooling is required.

To achieve even greater solid angle, novel designs using non-circular sensors, or clusters of small sensors have been introduced. By minimizing the sample to crystal distance, while retaining sensor area, solid angles about $3 \mathrm{x}$ higher than those achieved using conventional single circular sensors have been achieved. These very high solid angle SDDs for TEM are able to detect and map elements down to the atomic level in short analysis times, hence limiting effects of drift, beam damage and sample contamination.

In this study a $100 \mathrm{~mm}^{2}$ non-circular sensor achieving between 0.7 and $1.0 \mathrm{str}$ (depending on microscope geometry) has been designed with a windowless configuration. The windowless operation of this $\mathrm{X}-\mathrm{Max}^{\mathrm{N}}$ 100TLE detector shows a greatly enhanced sensitivity to light element $\mathrm{X}$-rays, and this is important for the analysis of semiconductors, polymers and biomaterials. Elements such as $\mathrm{N}$ and $\mathrm{O}$ are enhanced by up to $4 \mathrm{x}$ over very high solid angle SDD detectors that use an ultra-thin polymer window (Figure 1). The low energy capabilities play an important role in the discrimination of structures in semiconductors, such as between $\mathrm{Ti}$ and $\mathrm{TiN}$ layers, for ascertaining performance of these devices. Figure 2 shows Ti/TiN distribution in a semiconductor device. The composition of the layer around the W plug has been determined using 'TruMap' where peak deconvolution and background removal has been performed on the maps in real time. It can be clearly seen that the layer around the plug is TiN. However, it can also be seen that during assembly, Ti has diffused into the Al layer but no $\mathrm{N}$ is present.

Very large solid angle SDD detectors are important in the collection of atomic column data, where collection times may need to be limited to reduce the effects of sample drift, beam damage and contamination. Meaningful maps may be collected in a few minutes due to the high collection efficiency of these detectors. Figure 3 shows a layered image of CdTe collected in under 2 minutes.

In conclusion, it can be seen that very large solid angle windowless detectors extend the capabilities of EDS analysis to application areas which had formerly regarded as being limited to Electron Energy Loss Spectrometry (EELS). High collection efficiency, especially for low photon energies now makes EDS a viable option for light element analysis on the nanoscale.

[1] M. Watanabe and C.A. Wade, Microsc. Microanal., 19 (2013), Suppl. 2, Cambridge University Press,1264-1265 


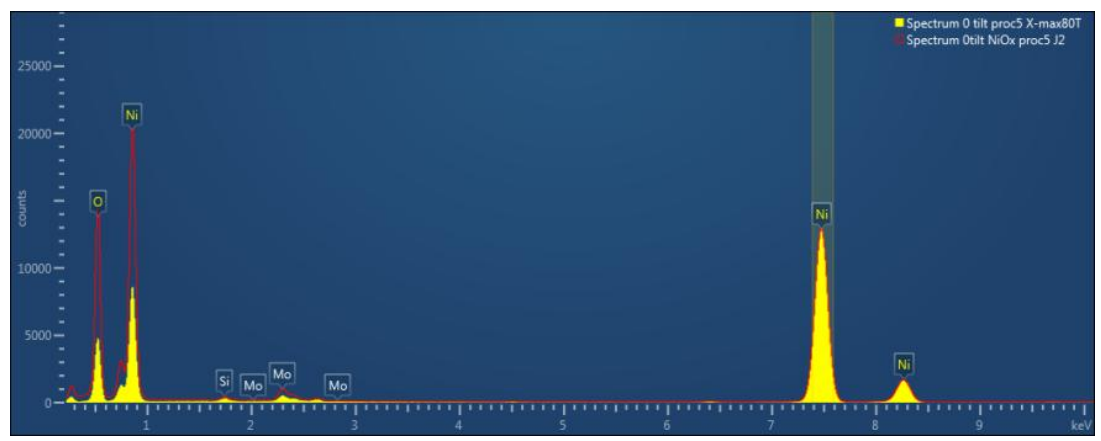

Figure 1. Comparison of X-Max 80T (yellow - 80mm² window) and $\mathrm{Max}^{\mathrm{N}}$ 100TLE (red - 100mm $\mathrm{m}^{2}$ very large solid angle windowless) SDDs. Spectrum has been normalized on the Ni Ka peak to show the sensitivity enhancement of the windowless operation for low energy X-rays

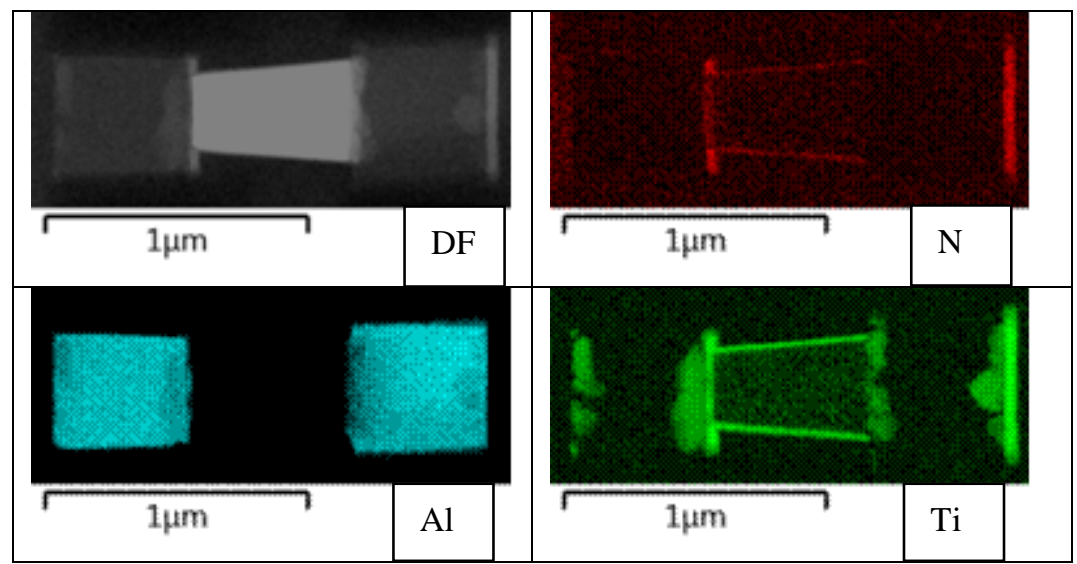

Figure 2. Dark field image of a W plug between two Al metal lines. X-ray maps of N and Ti show well defined TiN around $\mathrm{W}$ but Ti can be seen diffused into the Al with an absence of $\mathrm{N}$
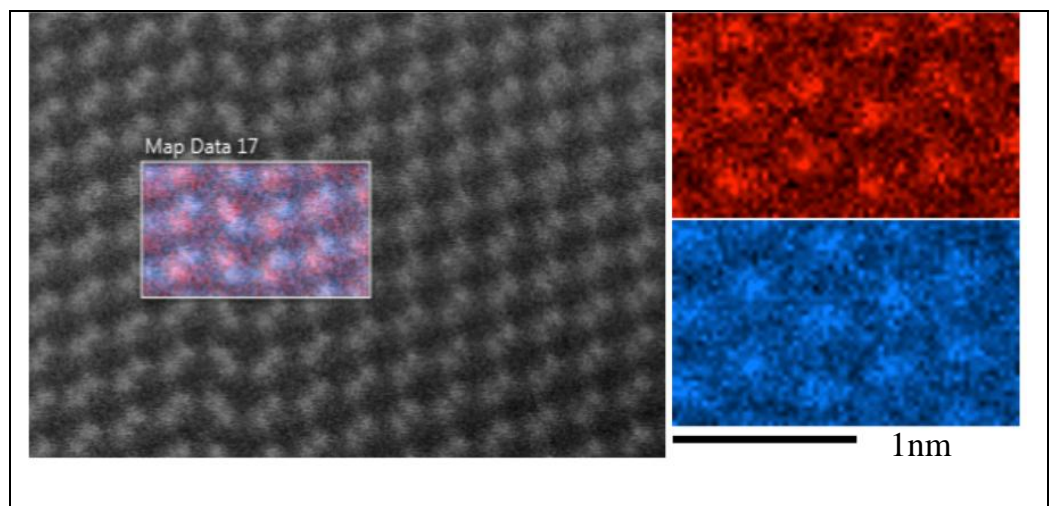

Figure 3. HAADF STEM image acquired across a twin boundary, viewed with the Cd L (red) and Te L (blue) X-ray maps overlaid. All data are raw and unbinned 